\title{
Correction: Redefining and measuring transplant conditioning intensity in current era: a study in acute myeloid leukemia patients
}

\begin{abstract}
Alexandros Spyridonidis (1) - Myriam Labopin - Bipin N. Savani (ib) Riitta Niittyvuopio - Didier Blaise (D) Charles Craddock - Gerard Socié $(\mathbb{D})$ Uwe Platzbecker - Dietrich Beelen - Noel Milpied - Jan J. Cornelissen • Arnold Ganser - Anne Huynh • Laimonas Griskevicius · Sebastian Giebel · Mahmoud Aljurf • Eolia Brissot • Florent Malard (1) - Jordi Esteve - Zinaida Peric · Frédéric Baron - Annalisa Ruggeri - Christoph Schmid • Maria Gilleece (1) - Norbert-Claude Gorin - Francesco Lanza - Roni Shouval • Jurjen Versluis · Gesine Bug • Yngvar Fløisand · Fabio Ciceri · Jamie Sanz $\mathbb{1 0} \cdot$ Ali Bazarbachi · Arnon Nagler · Mohamad Mohty
\end{abstract}

Published online: 6 March 2020

(c) Springer Nature Limited 2020

\section{Correction to: Bone Marrow Transplantation} https://doi.org/10.1038/s41409-020-0803-y published online 29 January 2020

In the original publication, the author Mahmoud Aljurf was omitted from the author list. This author's affiliation is
'Oncology Center, King Faisal Specialist Hospital \& Research Center, Riyadh, Saudi Arabia'. This has been corrected in both the PDF and HTML versions of the original article. 